\title{
Sophora tomentosa e Crotalaria vitellina (Fabaceae): biologia reprodutiva e interações com abelhas na restinga de Ubatuba, São Paulo
}

\author{
Vinícius Lourenço Garcia de Brito ${ }^{1,3}$, Mardiore Pinheiro ${ }^{2}$ \& Marlies Sazima ${ }^{2}$ \\ ${ }^{\text {I} P r o g r a m a ~ d e ~ P o ́ s-g r a d u a c ̧ a ̃ o ~ e m ~ B i o l o g i a ~ V e g e t a l, ~ D e p a r t a m e n t o ~ d e ~ B i o l o g i a ~ V e g e t a l, ~}$ \\ Instituto de Biologia, Universidade Estadual de Campinas - UNICAMP, \\ CP 6109, CEP 13083-970 Campinas, SP, Brasil \\ ${ }^{2}$ Departamento de Biologia Vegetal, Instituto de Biologia, \\ Universidade Estadual de Campinas - UNICAMP, \\ CP 6109, CEP13083-970 Campinas, SP, Brasil \\ ${ }^{3}$ Autor para correspondência: Vinícius Lourenço Garcia de Brito, e-mail: viniciusduartina@yahoo.com.br
}

BRITO, V.L.G., PINHEIRO, M. \& SAZIMA, M. Sophora tomentosa and Crotalaria vitellina (Fabaceae): reproductive biology and interactions with bees in the restinga of Ubatuba, São Paulo. Biota Neotrop. 10(1): http://www.biotaneotropica.org.br/v10n1/en/abstract?article+bn03310012010.

\begin{abstract}
The study of plant-pollinator interactions is a major tool for conservation biology of fragmented habitats like the Atlantic Rain Forest and for the study of evolutionary traits that rule these interactions. Information on reproductive biology and floral visitor interactions of Sophora tomentosa and Crotalaria vitellina (Fabaceae) is presented in this study. Both species are common in the restinga formation of the Atlantic Rain Forest in the Núcleo Picinguaba, Parque Estadual da Serra do Mar, Ubatuba - São Paulo. These species occur in the same areas, the flowering period is the same for both and they have yellow flowers that offer nectar as the major reward. Both are self-compatible, although fruit and seed set depend on the pollinators. Xylocopa brasilianorum and Megachile sp.1 were pollinators of S. tomentosa, whereas $C$. vitellina was pollinated by both the aforementioned bees and Bombus morio, Centris labrosa and two separate species of Megachile. All these bees have long tongues and are able to reach nectar at the bottom of the nectar chamber by legitimate visits. The inflorescences of $S$. tomentosa received more visits $(0,62$ visits/inflorescence/day) than the inflorescences of $C$. vitellina (0,37 visits/inflorescence/day). However, the fruit set of $S$. tomentosa (33\%) is similar to that of $C$. vitellina $(42 \%)$ in natural conditions, probably due to different pollinator efficiency. Having shorter tongues, Trigona and Augochlora bees have no access to the nectar chamber by means of legitimate visits. Hence, the dimensions of the nectar chamber of S. tomentos $a$ and $C$. vitellina act as a selective barrier to short-tongued bees, thus guaranteeing more nectar to the long-tongued visitors. These legume pollinators are very common in the restinga forest, and they act as pollen vectors of other species in this ecosystem as well. The preservation of these legume plants is important to the diversity of bees and essential to the plant community.
\end{abstract}

Keywords: bees, flowering phenology, Leguminosae, Atlantic Rainforest, nectariferous chamber, self-compatibility.

BRITO, V.L.G., PINHEIRO, M. \& SAZIMA, M. Sophora tomentosa e Crotalaria vitellina (Fabaceae): biologia reprodutiva e interações com abelhas na restinga de Ubatuba, São Paulo. Biota Neotrop. 10(1): http://www. biotaneotropica.org.br/v10n1/pt/abstract?article+bn03310012010.

Resumo: O conhecimento das interações entre plantas e seus polinizadores tem-se destacado como ferramenta na biologia da conservação de ambientes degradados, como a Mata Atlântica e no estudo da evolução de características morfológicas que medeiam estas interações. Neste estudo são apresentadas informações sobre a biologia reprodutiva e as interações com os visitantes florais de Sophora tomentosa e Crotalaria vitellina (Fabaceae), espécies comuns em áreas de restinga na Mata Atlântica no Núcleo Picinguaba, Parque Estadual da Serra do Mar, Ubatuba - São Paulo. Estas espécies são sincronopátricas, possuem flores amarelas dispostas em racemos e oferecem néctar como principal recurso. Ambas as espécies são autocompatíveis, mas dependem de polinizadores para formação de frutos e sementes. Xylocopa brasilianorum e Megachile sp. 1 foram os polinizadores de $S$. tomentosa, ao passo que C. vitellina, além destas espécies, também foi polinizada por Bombus morio, Centris labrosa e mais duas espécies de Megachile. Estas espécies de abelhas possuem comprimento da língua compatível com as dimensões das câmaras nectaríferas, acessando o néctar por visitas legítimas. As inflorescências de $S$. tomentosa foram mais visitadas ( 0,62 visitas/inflorescência/dia) do que as de $C$. vitellina $(0,37$ visitas/inflorescência/dia). Entretanto, em condições naturais, a frutificação em $S$. tomentosa $(33 \%)$ é semelhante à de $C$. vitellina $(42 \%)$, provavelmente devido às diferenças na eficiência de cada polinizador. Espécies de Trigona e de Augochlora não têm acesso ao néctar em visitas legítimas, pois não possuem comprimento da língua compatível com as dimensões das câmaras nectaríferas. Portanto, as dimensões das câmaras nectaríferas de $S$. tomentosa e $C$. vitellina funcionam como barreira seletiva às espécies de abelhas com língua curta, assegurando maior oferta de néctar aos polinizadores. Ainda, os polinizadores destas leguminosas são comuns na restinga e atuam como vetores de pólen de diversas espécies neste ecossistema. Portanto a manutenção destas leguminosas é importante para a diversidade da fauna de abelhas e essencial para a comunidade de plantas.

Palavras-chave: abelhas, fenologia da floração, Leguminosae, Mata Atlântica, câmara nectarífera, autocompatibilidade. 


\section{Introdução}

Estudos sobre a biologia da reprodução têm sido utilizados para a conservação e recuperação de habitats naturais afetados pela fragmentação, pois podem fornecer informações importantes relacionadas à facilitação e competição por polinizadores, sucesso reprodutivo e manutenção do fluxo gênico intraespecífico (Kearns et al. 1998, Alves-dos-Santos 2003, Ghazoul 2006). Por outro lado, estudos sobre a biologia da polinização podem contribuir para o conhecimento da biodiversidade, bem como para o entendimento da evolução das interações planta-polinizador (Price 2002). Neste último caso, os animais tendem a aperfeiçoar a coleta de recursos como pólen e néctar, enquanto que as plantas tendem a otimizar a transferência e recepção do pólen, buscando o menor custo energético possível (Pellmyr 2002).

A morfologia floral é um dos aspectos mais importantes nas interações planta-polinizador, pois determina a acessibilidade ao néctar, a eficiência de deposição de pólen no corpo do polinizador e a eficiência de aquisição de pólen pelo estigma (Sakai et al. 1999). A fenologia da floração também pode contribuir para a compreensão da dinâmica das interações planta-polinizadores em comunidades (Newstrom et al. 1994). Períodos de floração similares, por exemplo, e partilha de polinizadores, podem afetar o sucesso reprodutivo das plantas (Rathcke \& Lacey 1985), bem como influenciar a dinâmica das populações de visitantes florais.

A família Fabaceae é a terceira maior família entre as angiospermas e a segunda família mais importante economicamente (Judd et al. 2009). Várias espécies desta família desempenham papel vital na biogeoquímica global, por apresentarem nódulos com bactérias fixadoras de nitrogênio atmosférico (Sprent 2001). No Brasil, esta família está representada por cerca de 190 gêneros e 2.100 espécies, que possuem papel de destaque como elemento florístico em diversas formações vegetais, principalmente daquelas pertencentes ao domínio atlântico, onde possuem destacada importância por sua riqueza e abundância (Lima 2000).

Diante da grande riqueza e importância ecológica dessa família no Brasil, ainda existem poucos estudos sobre biologia da polinização e da reprodução sobre espécies de Fabaceae (cf. Lopes \& Machado 1996, Gibbs \& Sassaki 1998, Gibbs et al. 1999a, b, Prata de Assis Pires \& Freitas 2008, Lewis \& Gibbs 1999, Freitas \& Oliveira 2002, Carvalho \& Oliveira 2003, Westerkamp 2004, Agostini et al. 2006, Nogueira \& Arruda 2006). O gênero Sophora L. contém cerca de 50 espécies representadas por ervas perenes, arbustos e árvores distribuídas principalmente na Europa e Ásia, sendo amplamente introduzidas na África, com 3-4 espécies no oeste da América do Sul (Chile, Ilhas Juan Fernandez e Argentina,) e S. tomentosa L. na região costeira dos paleotrópicos e do leste do Brasil (Pennington et al. 2005). Pouco se conhece sobre a polinização e a biologia reprodutiva deste gênero, com exceção de estudos sobre S. tomentosa (Nogueira \& Arruda 2006), que é polinizada por abelhas e é autocompatível e S. fernandeziana, que é polinizada por aves e há possibilidade de ocorrer um mecanismo de autoincompatibilidade ovariana (Bernardello et al. 2004). Crotalaria L. é um gênero com cerca de 690 espécies e que inclui ervas perenes, arbustos e subarbustos principalmente nos trópicos do Velho Mundo, havendo 59 espécies endêmicas e outras 15 introduzidas no Novo Mundo e cerca de 35 espécies na América do Sul, principalmente no Brasil (Van Wyk 2005). As poucas informações sobre biologia da polinização e reprodução em espécies de Crotalaria correspondem a C. retusa (Jacobi 2005), C. micans, C. stipularia (Etcheverry et al. 2003) e C. juncea (Free 1970) indicam que estas espécies são polinizadas por abelhas, mas são autocompatíveis e pode ocorrer autopolinização espontânea.
Foi realizado um estudo comparativo sobre a biologia reprodutiva e as interações com as abelhas visitantes de Sophora tomentosa e Crotalaria vitellina. Estas leguminosas são arbustos que apresentam flores morfologicamente semelhantes e são comuns em áreas de restinga, sendo suas populações sincronopátricas na região de Ubatuba, São Paulo. Os principais objetivos foram responder às perguntas: a) qual é o padrão de floração de $S$. tomentosa e $C$. vitellina nessa região?, b) quais atributos da biologia e morfologia floral são semelhantes entre as espécies?, c) qual é o sistema de reprodução e há dependência de vetores de pólen para a formação de frutos?, d) estas leguminosas apresentam as mesmas espécies de polinizadores?, e) existem atributos da morfologia floral que dirigem a interação planta-polinizador?

\section{Material e Métodos}

O presente estudo foi desenvolvido no Parque Estadual da Serra do Mar, Núcleo de Desenvolvimento Picinguaba (NDP), em Ubatuba ( $23^{\circ} 22^{\prime} \mathrm{S}$ e $\left.44^{\circ} 48^{\prime} \mathrm{O}\right)$, São Paulo. A área de estudo localiza-se na planície costeira, próxima à orla da Praia da Fazenda, e caracteriza-se como floresta de restinga (Bencke \& Morellato 2002). O clima da região é tropical chuvoso, apresentando uma estação superúmida de outubro a abril, com precipitação média superior a $200 \mathrm{~mm}$ mensais, e uma estação menos úmida de maio a setembro, com precipitação média acima de $100 \mathrm{~mm}$ mensais (Morellato et al. 2000). A precipitação média anual é de $2.100 \mathrm{~mm}$ e a temperatura média anual é cerca de $22{ }^{\circ} \mathrm{C}$ (Bencke \& Morellato 2002).

A floração das espécies foi acompanhada mensalmente, entre os meses de setembro de 2006 a agosto de 2007, tendo-se considerado como pico de floração o mês em que o número máximo de indivíduos ( $n=150$ para cada espécie) apresentou a fenofase. Observações fenológicas preliminares e dados de Romera (1999) também foram considerados para definição dos padrões de floração.

O comprimento das inflorescências $(n=12$ indivíduos de C. vitellina e $\mathrm{n}=5$ indivíduos de $S$. tomentosa), o número de inflorescências por indivíduo, bem como o número de flores abertas por inflorescência ( $\mathrm{n}=25$ indivíduos de cada espécie) foi verificado em campo. Os eventos da antese como: horário de abertura das flores, deiscência das anteras, receptividade do estigma e emissão de odor foram verificados diretamente em campo $(n=10$ flores, $\mathrm{n}=5$ indivíduos de cada espécie). A presença de osmóforos foi testada ( $\mathrm{n}=10$ flores, $\mathrm{n}=5$ indivíduos de cada espécie) com vermelho neutro e a receptividade estigmática com o método de atividade peroxidásica (Dafni 1992).

Medidas das estruturas florais foram feitas em laboratório, utilizando-se 15 flores fixadas em álcool 70\% ( $\mathrm{n}=10$ indivíduos de cada espécie). A concentração e o volume do néctar disponível (standing crop - flores não ensacadas) e acumulado (flores ensacadas) foram verificados em flores recém abertas com o uso de microsseringa e refratômetro manual (Galetto \& Bernardello 2005), ao longo de um dia, a cada duas horas das 08:00 às 16:00 $(\mathrm{n}=3$ indivíduos para cada intervalo: 30 flores de cada espécie, sendo 15 ensacadas e 15 não ensacadas).

A viabilidade polínica foi verificada com carmim acético ( $\mathrm{n}=5$ flores, $\mathrm{n}=5$ indivíduos) e o sistema reprodutivo através de testes de agamospermia (flores emasculadas $-n=20$ ), autopolinização espontânea $(n=20)$, autopolinização manual $(n=20)$ e polinização cruzada (flores emasculadas $-\mathrm{n}=17$ ), usando flores ensacadas na fase de botão para cada espécie (Radford et al. 1974). Outras flores ( $\mathrm{n}=30$ para $S$. tomentosa $\mathrm{e} \mathrm{n}=36$ para $C$. vitellina) foram marcadas para estimar a frutificação em condições naturais. O desenvolvimento de tubos polínicos foi verificado em microscópio de fluorescência em flores autopolinizadas manualmente e de polinizações cruzadas, fixa- 
das em FAA 50\%, nos intervalos de 12, 24, 36, 48 e 72 horas após as polinizações ( $\mathrm{n}=3$ para cada intervalo, $\mathrm{n}=10$ indivíduos), amolecidas e clareadas em água sanitária (adaptado de Martin 1959).

A freqüência dos visitantes florais de cada espécie foi calculada através de observações focais, registrando-se o número de visitas por inflorescência ( $\mathrm{n}=8$-20 inflorescências) em intervalos de 30 minutos, das 07:00 às 16:00, em seis dias não consecutivos, totalizando 27 horas de observações para cada espécie. Os polinizadores foram definidos através da análise do seu comportamento (contato com elementos sexuais) durante as visitas, de análises de fotografias dos visitantes nas flores e da correspondência entre a morfologia e tamanho do corpo com a morfologia e tamanho das estruturas reprodutivas das flores. Medidas do comprimento do corpo e da língua das abelhas visitantes foram tomadas em laboratório com o uso de um paquímetro. Espécimes das abelhas e exsicatas das plantas foram depositadas, respectivamente, no Museu de Zoologia (ZUEC) e no herbário (UEC) da Universidade Estadual de Campinas (UNICAMP).

Atributos dos indivíduos, inflorescências e flores das duas espécies foram comparados pelo teste t ou, quando não comprovada a normalidade dos dados pelo teste de Shapiro-Wilk, esses dados foram comparados pelo teste U de Mann-Whitney. A comparação entre o

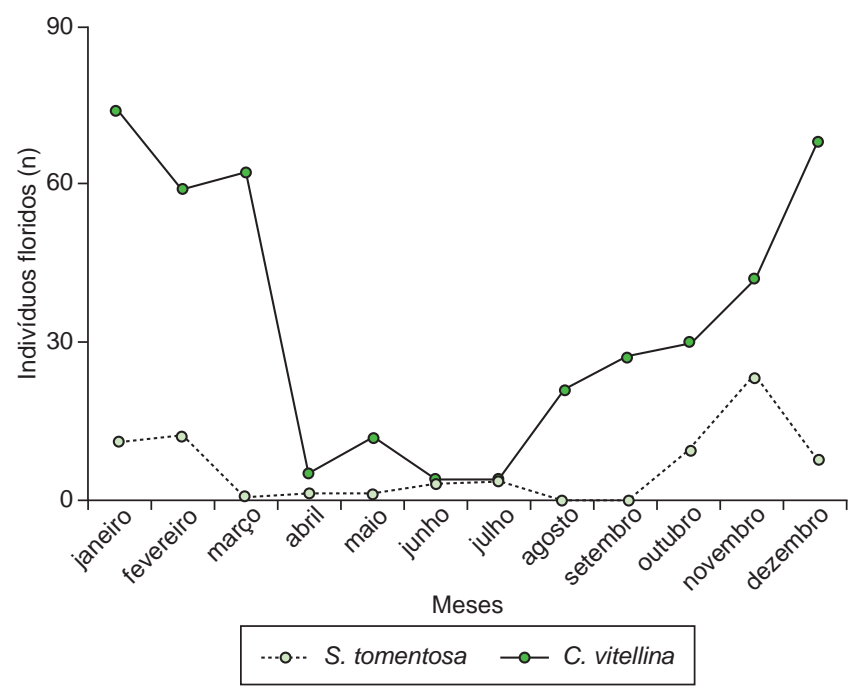

Figura 1. Número de indivíduos floridos das populações de Sophora tomentosa e Crotalaria vitellina estudadas na restinga de Picinguaba, Ubatuba, SP. $(\mathrm{n}=150)$

Figure 1. Number of flowering plants of Sophora tomentosa and Crotalaria vitellina at restinga of Picinguaba, Ubatuba, SP. $(\mathrm{n}=150)$. número de frutos produzidos por ambas as espécies em condições naturais foi feita através de teste Qui-quadrado de Pearson. O número de visitas/inflorescência/dia entre as espécies e os atributos das abelhas e das flores não foram comparados devido ao baixo número de repetições. Para todos os testes foi assumido um intervalo de confiança de $95 \%$ e foi utilizado o software Systat11 (Systat Software Inc., Point Richmond, CA).

\section{Resultados}

Sophora tomentosa e C. vitellina apresentam, respectivamente, padrão de floração subanual e contínuo (sensu Newstrom et al. 1994), sendo os meses mais úmidos e quentes do ano o período com maior número de indivíduos floridos. O pico de floração de $S$. tomentosa foi em novembro e o de C. vitellina em janeiro (Figura 1). Estas espécies são sincronopátricas havendo sobreposição do período de floração de seis a oito meses, porém, $S$. tomentosa apresentou menor quantidade de indivíduos floridos que $C$. vitellina ao longo deste período (Figura 1).

Os indivíduos de $S$. tomentosa possuem mais inflorescências que os de C. vitellina (Tabela 1). Além disso, S. tomentosa possui inflorescências maiores e mais vistosas e apresentam mais flores abertas por dia que $C$. vitellina (Tabela 1, Figura 2 a,b). Em ambas as espécies as inflorescências são racemos e as flores papilionadas, de cor amarela (Figura 2 a,b), diurnas e ligeiramente perfumadas em $S$. tomentosa (há osmóforos nas laterais inferiores das alas), fornecem néctar como principal recurso. Em S. tomentosa os estames são livres, mas soldados na base formando um pequeno tubo e o acesso ao néctar é feito pela parte livre dos estames. Por outro lado, em C. vitellina os estames são monadelfos, formando um tubo mais longo e o néctar é acessado por uma pequena abertura (ver Figura 2b, D em Etcheverry et al. 2003). Nestas espécies a entrada ao néctar é coberta pela unguícula do estandarte. Tanto as flores como os elementos reprodutivos são maiores em $S$. tomentosa do que em $C$. vitellina, porém as câmaras nectaríferas possuem tamanho similar (Tabela 1). A viabilidade polínica em ambas as espécies é alta, sendo $97 \%$ em S. tomentosa e $99 \%$ em C. vitellina.

O volume de néctar acumulado de $S$. tomentosa é de $9 \mu \mathrm{l}$ em parte da manhã e ao longo do dia se mantém mais ou menos estável (4-5 $\mu 1)$, diminuindo para cerca de $2 \mu$ laté o final do dia; o volume de néctar disponível (standing crop) também é relativamente maior no início da manhã, mas diminui consideravelmente e se mantém estável até o final do dia (Figura 3). O volume de néctar acumulado por $C$. vitellina é baixo $(3 \mu \mathrm{l})$ se comparado com $S$. tomentosa, permanecendo quase inalterado durante o período da manhã, com ligeira redução no período da tarde e o volume de néctar disponível apresenta um pico (cerca de $5 \mu \mathrm{l}$ ) no meio da manhã, diminuindo após as 10 horas, mantendo

Tabela 1. Características florais (dimensões em mm) de Sophora tomentosa e Crotalaria vitellina na restinga de Picinguaba, Ubatuba, SP. T - teste t; U - teste de Mann-Whitney; g.1.-graus de liberdade; * $\mathrm{p}<0,05$; *** $\mathrm{p}<0,001$; NS - não significativo.

Table 1. Floral characteristics (dimensions in $\mathrm{mm}$ ) of Sophora tomentosa and Crotalaria vitellina in the restinga of Picinguaba, Ubatuba, SP. $\mathrm{T}-\mathrm{t}$ test; $\mathrm{U}-$ Mann-Whitney test; g.1.- degrees of freedom; * $\mathrm{p}<0,05 ; * * * \mathrm{p}<0,001$; NS - not significant.

\begin{tabular}{|c|c|c|c|c|c|}
\hline Atributos & Sophora tomentosa & Crotalaria vitellina & teste & g.l. & $\mathbf{p}$ \\
\hline Inflorescências/indivíduo & $1,8( \pm 0,96)$ & $3,8( \pm 2,15)$ & $\mathrm{U}=512$ & 1 & $* * *$ \\
\hline Flores /inflorescência & $2,3( \pm 1,90)$ & $1,2( \pm 0,45)$ & $\mathrm{U}=156$ & 1 & $* * *$ \\
\hline Inflorescência (compr.) & $22,176( \pm 6,395)$ & $12,083( \pm 5,587)$ & $\mathrm{T}=-3,074$ & 6,7 & $*$ \\
\hline Estandarte (diâm.) & $13,54( \pm 1,252)$ & $10,95( \pm 1,034)$ & $\mathrm{T}=-6,197$ & 27 & $* * *$ \\
\hline Flor (compr.) & $21,65( \pm 0,792)$ & $11,75( \pm 0,655)$ & $\mathrm{U}=0$ & 1 & $* * *$ \\
\hline Gineceu (compr.) & $17,56( \pm 1,285)$ & $10,01( \pm 0,547)$ & $\mathrm{U}=0$ & 1 & $* * *$ \\
\hline Estame (compr.) & $19,42( \pm 0,740)$ & $7,93( \pm 0,919)$ & $\mathrm{U}=0$ & 1 & $* * *$ \\
\hline Câmara nectarífera (compr.) & $3,30( \pm 0,362)$ & $3,22( \pm 0,341)$ & $\mathrm{T}=-0,613$ & 27,9 & NS \\
\hline
\end{tabular}



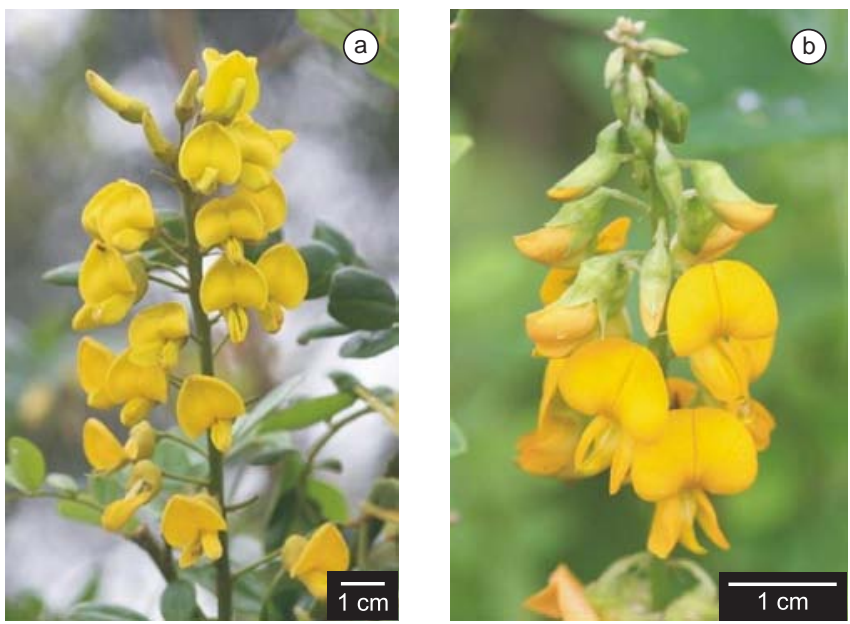

Figura 2. Aspecto geral das inflorescências de a) Sophora tomentosa; e b) Crotalaria vitellina ocorrentes na restinga de Picinguaba, Ubatuba, SP.

Figure 2. Inflorescences of a) Sophora tomentosa; and b) Crotalaria vitellina occurring at restinga of Picinguaba, Ubatuba, SP.

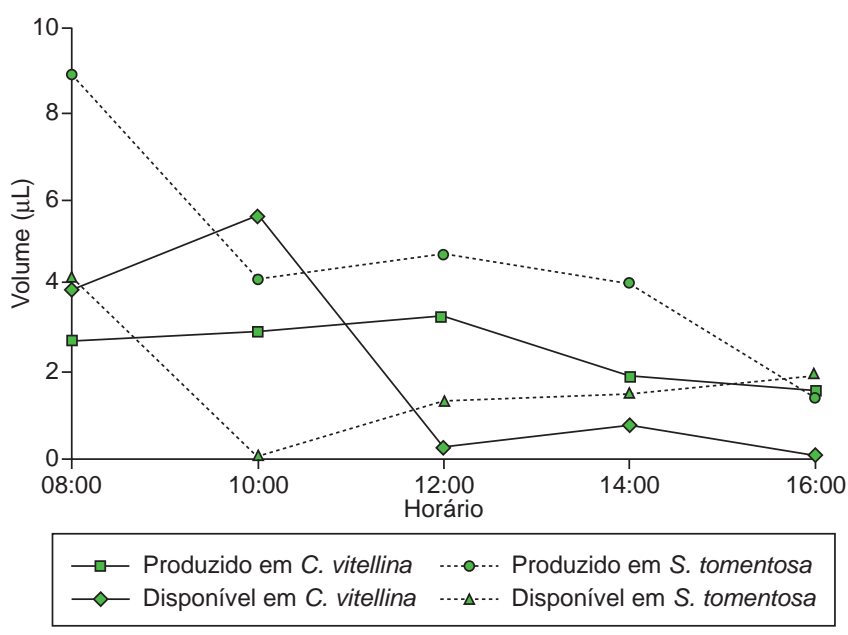

Figura 3. Néctar produzido e disponível em Sophora tomentosa e Crotalaria vitellina ao longo do dia na restinga de Picinguaba, Ubatuba, SP. $(\mathrm{n}=3$ por intervalo).

Figure 3. Accumulated and standing crop nectar in Sophora tomentosa and Crotalaria vitellina at restinga of Picinguaba, Ubatuba, SP. $(n=3$ per interval).

valores muito baixos até ao final da tarde (Figura 3). A concentração do néctar variou de $16,0-32,0 \%(26,7 \pm 5,74)$ em $S$. tomentosa e $17,0-32,0 \%(27,7 \pm 3,66)$ em C. vitellina.

Os resultados dos tratamentos de polinização indicam que as espécies não se reproduzem por agamospermia e nem por autopolinização espontânea, mas são autocompatíveis (Tabela 2). Em condições naturais a produção de frutos em $S$. tomentosa é semelhante à de $C$. vitellina $\left(\chi^{2}=0,483 ;\right.$ g.l. $\left.=3 ; \mathrm{p}=0,487\right)$ (Tabela 2). $\mathrm{O}$ desenvolvimento de tubos polínicos em $S$. tomentosa é mais rápido do que em $C$. vitellina, tanto em polinizações cruzadas como em autopolinizações. Em polinizações cruzadas de $S$. tomentosa, após 12 horas os tubos polínicos alcançam a base do estilete, após 24 horas atingem a região do ovário e após 72 horas não são mais visíveis, provavelmente degeneraram. Assim a fertilização possivelmente ocorre entre 24 e 48 horas após a polinização. Em C. vitellina os
Tabela 2. Percentual de frutos de Sophora tomentosa e Crotalaria vitellina em tratamentos sobre o sistema de reprodução na restinga de Picinguaba, Ubatuba, SP. (frutos/flores). Números com mesma letra indicam que não há diferença significativa.

Table 2. Percentage of fruit set of Sophora tomentosa and Crotalaria vitellina in different breeding system treatments in the restinga of Picinguaba, Ubatuba, SP. (fruits/flowers). Numbers with the same letter show that there is no significant difference.

\begin{tabular}{lcc}
\hline \multicolumn{1}{c}{ Tratamentos } & $\begin{array}{c}\text { Sophora } \\
\text { tomentosa }\end{array}$ & $\begin{array}{c}\text { Crotalaria } \\
\text { vitellina }\end{array}$ \\
\hline Agamospermia & $0 \%(0 / 20)$ & $0 \%(0 / 20)$ \\
Autopolinização manual & $15 \%(3 / 20)$ & $40 \%(8 / 20)$ \\
Autopolinização espontânea & $0 \%(0 / 20)$ & $0 \%(0 / 20)$ \\
Polinização cruzada & $0 \%(0 / 17)$ & $18 \%(3 / 17)$ \\
Controle (condições naturais) & $33 \%(10 / 30)^{\mathrm{a}}$ & $42 \%(15 / 36)^{\mathrm{a}}$ \\
\hline
\end{tabular}

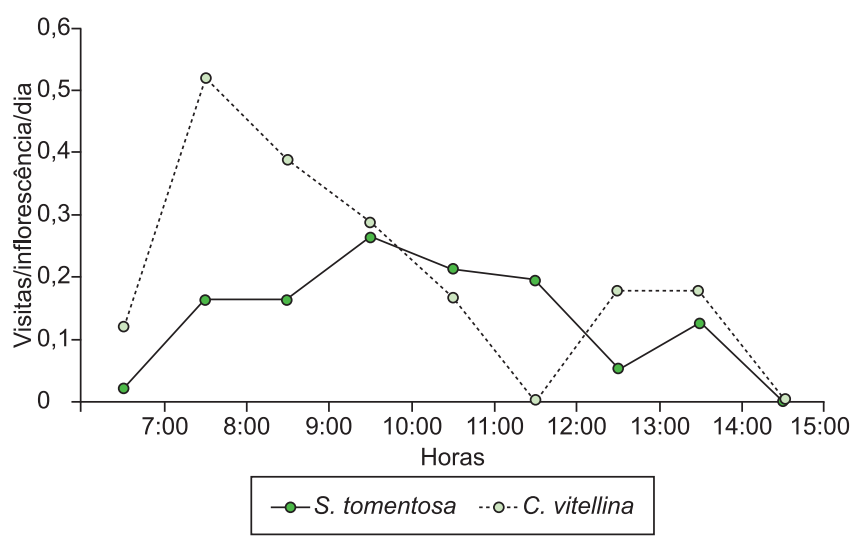

Figura 4. Freqüência de visitas de abelhas às inflorescências de Sophora tomentosa e Crotalaria vitellina ao longo do dia na restinga de Picinguaba, Ubatuba, SP.

Figure 4. Visitors frequency on the inflorescences of Sophora tomentosa and Crotalaria vitellina at restinga of Picinguaba, Ubatuba, SP.

grãos germinam somente após 12 horas, os tubos atingem a região terminal do estilete após 48 horas e a fertilização provavelmente ocorre entre 48 e 72 horas.

As visitas de abelhas em $S$. tomentosa ocorrem principalmente no início do dia, enquanto que em $C$. vitellina se distribuem ao longo do dia, mas em ambas as espécies a quantidade de visitas é baixa no período da tarde (Figura 4). Dentre os polinizadores, Xylocopa brasilianorum e Megachile sp.1 (Figura 5 a-c) foram os únicos compartilhados pelas duas espécies, mas $C$. vitellina ainda foi polinizada por Bombus morio, Centris labrosa e mais duas espécies de Megachile (Tabela 3). Estas espécies de abelhas possuem comprimento da língua acima de 4,0 mm, dimensão apropriada para acessar o néctar na câmara nectarífera através de visitas legítimas e comprimento do corpo acima de $8,5 \mathrm{~mm}$, tamanho adequado para contatar as estruturas reprodutivas das flores concomitantemente à tomada de néctar (Figura 6, Tabela 3). O comportamento dos polinizadores durante as visitas às flores das duas espécies foi similar. Para acessar o néctar as abelhas pousam sobre as alas e peças da quilha e em seguida inserem a cabeça na base do estandarte, deslocando-o e tornando acessível a entrada pela qual é inserida a língua para alcançar o néctar (Figura 5a-c). Durante esta atividade as alas e as peças da quilha são deslocadas pelas pernas, liberando as anteras e o estigma, ocasião em que contatam a região ventral do tórax em Bombus e Xylocopa (Figura 5a-b) ou do abdômen em Centris e Megachile (Figura 5 c). A taxa de visitas de polinizadores às inflorescências de $S$. tomentosa 

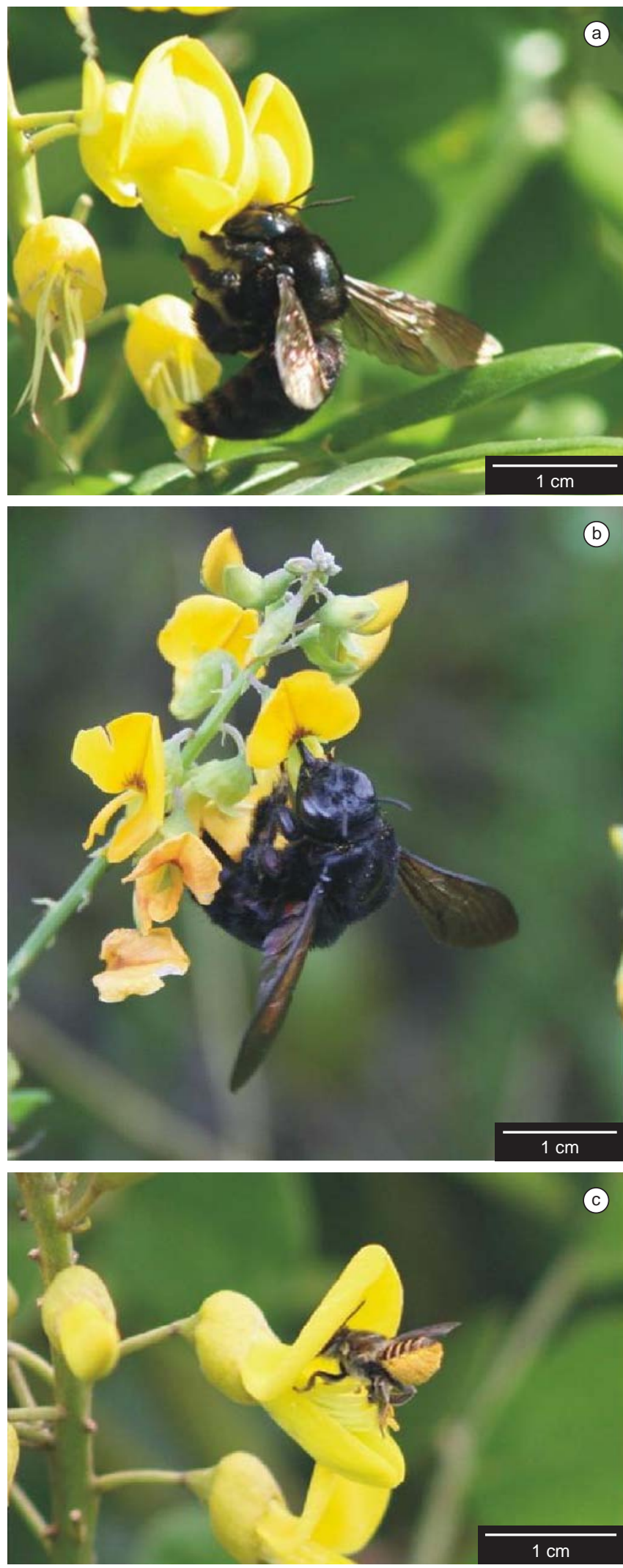

Figura 5. Xylocopa brasilianorum em visita a (a) S. tomentosa e (b) C. vitellina e (c) Megachile sp. 1 em visita a S. tomentosa.

Figure 5. Xylocopa brasilianorum visiting (a) S. tomentosa and (b) C. vitellina and (c) Megachile sp. 1 visiting S. tomentosa.
Tabela 3. Dimensões (mm) corpóreas das espécies de abelhas que visitaram as flores de Sophora tomentosa e Crotalaria vitellina na restinga de Picinguaba, Ubatuba, SP. $(\mathrm{n}=1-6)$. pol = polinizador; pil = pilhador

Table 3. Body dimensions ( $\mathrm{mm}$ ) of the floral visitors of Sophora tomentosa and Crotalaria vitellina in the restinga of Picinguaba, Ubatuba, SP. $(n=1-6)$. pol $=$ pollinator; pil $=$ thief.

\begin{tabular}{lcccc}
\hline \multicolumn{1}{c}{ Espécies } & $\begin{array}{c}\text { Corpo } \\
\text { (compr.) }\end{array}$ & $\begin{array}{c}\text { Língua } \\
\text { (compr.) }\end{array}$ & $\begin{array}{c}\text { Sophora. } \\
\text { tomentosa }\end{array}$ & $\begin{array}{c}\text { Crotalaria. } \\
\text { vitellina }\end{array}$ \\
\hline $\begin{array}{l}\text { Xylocopa } \\
\text { brasilianorum }\end{array}$ & 20,31 & 10,43 & pol & pol \\
Bombus morio & 15,82 & 11,21 & - & pol \\
Centris labrosa & 13,23 & 6,06 & - & pol \\
Megachile sp.3 & 11,34 & 5,71 & - & pol \\
Megachile sp.1 & 10,81 & 5,44 & pol & pol \\
Megachile sp.2 & 8,98 & 4,10 & - & pol \\
Trigona sp.1 & 6,33 & 2,67 & - & pil \\
Trigona sp.3 & 6,34 & 1,83 & pil & - \\
Augochlora sp.1 & 6,29 & 2,52 & - & pil \\
Trigona sp.2 & 4,78 & 2,38 & - & pil \\
\hline
\end{tabular}

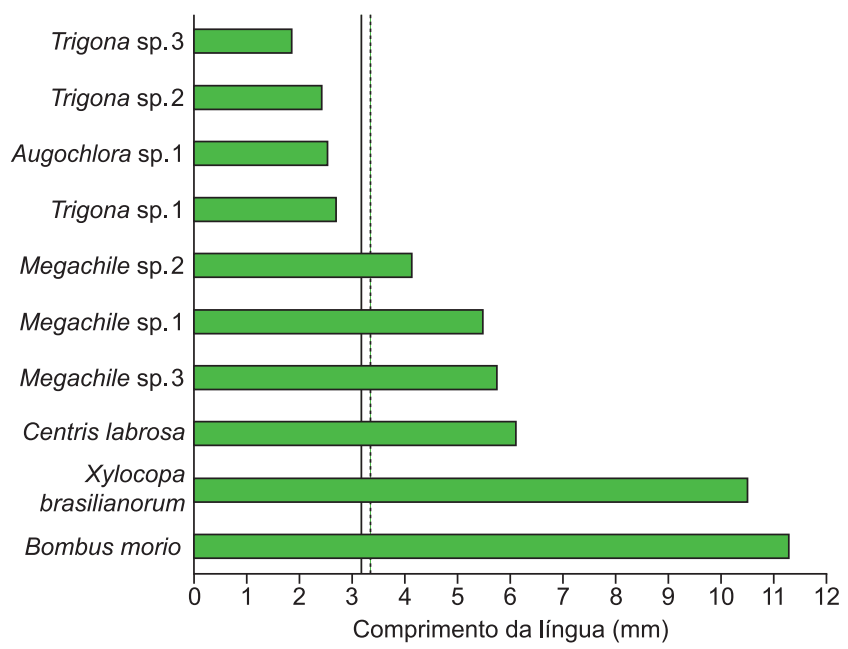

Figura 6. Comprimento das glossas dos visitantes florais de Sophora tomentosa e Crotalaria vitellina na restinga de Picinguaba, Ubatuba, SP. A linha tracejada e a sólida indicam o comprimento da câmara nectarífera das flores de $S$. tomentosa e $C$. vitellina, respectivamente.

Figure 6. Tongue length of flower visitors of Sophora tomentosa and Crotalaria vitellina at restinga of Picinguaba, Ubatuba, SP. The dotted and solid lines show the nectar chamber length of Sophora tomentosa and Crotalaria vitellina, respectively.

foi 0,62 visitas/inflorescência/dia e para C. vitellina foi 0,37 visitas/ inflorescência/dia.

Outros visitantes foram registrados em S. tomentosa e C. vitellina. Espécies de Trigona que possuem língua com comprimento inferior a 3,0 mm (Figura 6, Tabela 3), obtiveram néctar apenas através de visitas ilegítimas, furando a base do cálice. Estas espécies também visitaram as flores em busca de pólen, porém, devido ao pequeno tamanho corporal e ao fato de manipularem as anteras individualmente, contataram o estigma apenas esporadicamente. Augochlora sp.1 também possui língua $<3,0 \mathrm{~mm}$ (Figura 6, Tabela 3) e visitou as flores somente em busca de pólen, raramente contatando o estigma. Em $S$. tomentosa ainda foram observadas visitas irregulares do passeriforme Coereba flaveola (Coerebidae), mas que não contatou os elementos reprodutivos. 


\section{Discussão}

O padrão subanual e o período do pico de floração principal de $S$. tomentosa coincidem com o verificado por Nogueira \& Arruda (2006) para uma população dessa espécie na restinga de Santa Catarina, indicando que estas características sejam de cunho filogenético. O padrão de floração contínuo de $C$. vitellina parece ser característico das populações de restinga, uma vez que difere de populações em área de cerrado (Batalha \& Mantovani 2000) provavelmente devido às diferenças no regime de chuvas (Morellato et al. 2000). Este padrão de floração e a maior quantidade de indivíduos floridos de C. vitellina em relação aos de $S$. tomentosa provavelmente explica a riqueza de espécies de abelhas visitantes de $C$. vitellina. Além disso, a florada longa e a sobreposição com $S$. tomentosa representam recursos previsíveis, favorecendo a manutenção das populações de abelhas ao longo de boa parte do ano (Machado \& Sazima 2007).

Ambas as espécies possuem atributos florais semelhantes e característicos da síndrome de melitofila (Faegri \& Van Der Pijl 1979). Esta síndrome de polinização é predominante nesses gêneros (Arroyo 1981), embora haja espécies de Sophora polinizadas por aves e, nestes casos, com volume de néctar maior (Bernardello et al. 2004). Embora os dados de néctar sejam referentes a um dia de coleta, os valores registrados em flores recém abertas de $C$. vitellina são semelhantes aos encontrados para $C$. micans (Etcheverry et al. 2003). A diferença dos valores de néctar acumulado (3 ul) e do néctar disponível (5 ul) e a pouca variação na concentração em $C$. vitellina pode ser devido à sua reabsorção, como ocorre em $S$. fernandeziana (Bernardello et al. 2004). Os valores de volume e concentração de néctar nas espécies estudadas são característicos de espécies melitófilas dos trópicos e de regiões temperadas (Opler 1983, Pyke \& Waser 1981). Segundo Torres \& Galleto (1998), o padrão de apresentação de néctar está relacionado à sua produção e à taxa de visitas. Esse parece ser o caso das espécies estudadas, uma vez que as visitas por inflorescência (Figura 4) e o néctar acumulado e o disponível (standing crop) (Figura 3) indicam que em $S$. tomentosa o pico de visitas pela manhã corresponde ao pico de produção e disponibilidade de néctar, mas ao longo do dia ocorre diminuição do néctar e conseqüentemente das visitas e em $C$. vitellina a quantidade de néctar acumulado é quase constante ao longo do dia, assim como as visitas. Relação semelhante foi encontrada em S. fernandeziana (Bernardello et al. 2004). Porém, além da produção e da taxa de visitas, as diferenças filogenéticas e, principalmente, fatores históricos e ambientais também podem influenciar a dinâmica do néctar (Chalcoff et al. 2006).

Ausência de frutos por autopolinização espontânea provavelmente está relacionada ao não rompimento da cutícula estigmática à semelhança do que ocorre com C. juncea (Free 1970), indicando que essas espécies dependem de polinizadores para a formação de frutos. A ausência de frutos nos tratamentos de polinização cruzada em S. tomentosa e a menor produção em $C$. vitellina, quando comparada com a produção por autopolinizações manuais pode ser conseqüência de excesso de manipulação das flores durante os tratamentos (Jacobi et al. 2005) ou devido ao não rompimento da cutícula estigmática (Free 1970). A possibilidade de formar frutos por autopolinização (geitonogamia) via agentes, pode favorecer o sucesso reprodutivo na escassez de polinizadores (Endress 1994). Autocompatibilidade em S. tomentosa também foi verificada por Nogueira \& Arruda (2006), entretanto não havia essa informação para $C$. vitellina. Diversas espécies de leguminosas são autocompatíveis (Arroyo 1981), sendo essa característica também documentada para $S$. fernandeziana (Bernardello et al. 2004, mas veja abaixo), C. retusa (Jacobi et al. 2005) e C. micans (Etcheverry et. al. 2003). A semelhança na velocidade do crescimento dos tubos polínicos em tratamentos de autopolinização e de polinização cruzada nas duas espécies pode indicar que não há sítios de reações de autoincompatibilidade. Por outro lado, Bernardello et al. (2004) sugerem a ocorrência de sítios de autoincompatibilidade nos ovários de S. fernandeziana, mas aponta para a necessidade de mais experimentos, uma vez que o desenvolvimento de frutos não foi acompanhado. A velocidade de desenvolvimento dos tubos em C. vitellina é semelhante ao mencionado para C. micans por Etcheverry et al. (2003), sugerindo um padrão para este gênero.

Segundo Rathcke \& Lacey (1985) espécies simpátricas que possuem sobreposição de florada, semelhanças florais e atraem os mesmos polinizadores, podem ter sua reprodução favorecida ou não. Apesar de ambas as espécies compartilharem alguns polinizadores, os resultados indicam que $S$. tomentosa é mais visitada que C. vitellina. Esta diferença provavelmente está associada à maior quantidade de flores em $S$. tomentosa, o que poderia aumentar a atratividade (Mitchell et al. 2004). Além disso, os polinizadores tendem a visitar espécies com flores maiores ao invés de menores (Geber 1985) e visitar, preferencialmente, espécies que produzem mais néctar (Zimmerman 1988), ambas as características ocorrem nas flores de $S$. tomentosa. Entretanto, a frutificação em condições naturais é semelhante nas duas espécies, provavelmente devido às diferenças na eficiência de cada polinizador. O comportamento das abelhas durante as visitas nas flores de $S$. tomentosa é semelhante ao relatado por Nogueira \& Arruda (2006). Segundo Westerkamp (1997) flores do tipo quilha previnem que as abelhas coletem pólen ativamente durante a visita, e esta obtenção indireta de pólen garante que mais grãos sejam usados na polinização.

A relação entre as dimensões das línguas das espécies de abelhas e as dimensões das câmaras nectaríferas das flores de S. tomentosa e C. vitellina indica que esta funciona como barreira seletiva para as espécies de línguas curtas, assegurando maior oferta de néctar aos polinizadores. Segundo Pellmyr (2002) câmaras nectaríferas evoluíram por dois motivos: reduzir os efeitos negativos de parasitas ou polinizadores ineficientes que diminuem o sucesso reprodutivo; ou aumentar a eficácia dos polinizadores criando um caminho físico até o néctar e que garante a polinização. Esse aspecto é aplicável às câmaras das espécies estudadas, pois sua estrutura garante o melhor posicionamento dos polinizadores para que a transferência de pólen ocorra de forma eficiente. Entretanto, a atividade pilhadora das abelhas de línguas curtas pode interferir e diminuir as visitas dos polinizadores, cuja consequiência é a redução na quantidade de frutos produzidos (Sazima, I. \& Sazima, M. 1989).

Sophora tomentosa e C. vitellina são espécies abundantes na floresta de restinga e, além da sua importância ecossistêmica como leguminosas fixadoras de nitrogênio atmosférico, disponibilizam recursos para importantes vetores de pólen. As espécies de abelhas visitantes destas leguminosas são comuns na restinga e atuam como polinizadores de diversas outras espécies neste ambiente (Pansarin et al. 2008, Pinheiro \& Sazima 2007), portanto a manutenção destas interações é imprescindível para a conservação deste ecossistema.

\section{Agradecimentos}

A Iara F. Bressan pelo apoio técnico e a Aline L. Lima, Carlos E. P. Nunes, Gastão B. Rodrigues, Caio Migueli, Renato O. Santos, Danielle P. Maia, Marcelo M. Egea, Mário M. R. Cardoso pelo auxílio no campo, ao Graham E. Wyatt pela revisão do abstract, legendas e tabelas, ao Mauricio F. Otárola pela leitura crítica do manuscrito e aos assessores pelas valiosas sugestões. Ao CNPq pela bolsa de Iniciação Científica para VLGB e de produtividade em pesquisa para MS e à FAPESP pela bolsa de pós-doutorado para MP. Este trabalho também foi financiado pela Fundação de Amparo à Pesquisa do Estado de São Paulo (FAPESP) no âmbito do Projeto Temático Gradiente Funcional (Processo 03/12595-7), que faz parte do Programa BIOTA/FAPESP - 
O Instituto Virtual da Biodiversidade (www.biota.org.br). Autorização COTEC/IF 41.065/2005 e autorização IBAMA/CGEN 093/2005.

\section{Referências}

ALVES-DOS-SANTOS, I. 2003. Comunidade, conservação e manejo: o caso dos polinizadores. Rev. Tecnol. Ambient. 8(2):35-57.

AGOSTINI, K., SAZIMA, M. \& SAZIMA, I. 2006. Bird pollination of explosive flowers while foraging for nectar and caterpillars. Biotropica 38(5):674-678

ARROYO, M.T.K. 1981. Breeding systems and pollination biology in Leguminosae. In Advances in Legume Systematics: part 2. (R.M. Polhill \& P.H. Raven, eds). Royal Botanic Garden, Kew, p. 723-769.

BATALHA, M.A. \& MANTOVANI, W. 2000. Reproductive phenological patterns of Cerrado plant species at the Pé-de-Gigante Reserve (Santa Rita do Passa Quatro, SP, Brazil): a comparison between the herbaceous and woody floras. Rev. Bras. Biol. 60(1):129-145.

BENCKE, C.C. \& MORELLATO, P.L.C. 2002. Comparação de dois métodos de avaliação da fenologia de plantas, sua interpretação e representação. Rev. Bras. Bot. 25(3):269-275.

BERNARDELlO, G., AGUILAR, R. \& ANDERSON, G.F. 2004. The reproductive biology of Sophora fernandeziana (Leguminosae), a vulnerable endemic species from Isla Robinson Crusoe. Am. J. Bot. 91(2):198-206.

CARVALHO, D.A. \& OLIVEIRA, P.E. 2003. Biologia reprodutiva e polinização de Senna sylvestris (Vell.) Irwin \& Barneby (Leguminosae, Caesalpinioideae). Rev. Bras. Bot. 26(3): 319-328.

CHALCOFF, V.R., AIZEN, M.A., \& GALETTO L. 2006. Nectar concentration and composition of 26 species from the temperate forest of South America. Ann. Bot. 97(3):413-421.

DAFNI, A. 1992. Pollination ecology: a practical approach. Oxford University Press, Oxford.

ENDRESS, P.K. 1994. Diversity and evolutionary biology of tropical fkowers. Cambridge University Press, Cambridge.

ETCHEVERRY, A.V., PROTOMASTRO J.J. \& WESTERKAMP C. 2003. Delayed autonomous self-pollination in the colonizer Crotalaria micans (Fabaceae, Papilionoideae): structural and functional aspects. Plant Syst. Evol. 239(1-2):15-28.

FAEGRI, K. \& Van der PIJL, V. 1979. The principles of pollination ecology. Pergamon Press, Oxford.

FREE, J.B. 1970. Insect pollination of crops. Academic Press, New York.

FREITAS, C.V. \& OLIVEIRA, P.E. 2002. Biologia reprodutiva de Copaifera langsdorffii Desf. (Leguminosae-Caesalpinioideae). Rev. Bras. Bot. 25(3):311-321.

GALETTO, L. \& BERNARDELLO, G. 2005. Rewards in flowers: néctar. In Practical Pollination Biology (A. Dafni, P.G. Kevan \& B.C. Husband, eds). Enviroquest, Ontario, p. 261-313.

GHAZOUL, J. 2006. Floral diversity and the facilitation of pollination. J. Ecol. 94(2): 295-304.

GEBER, M.A. 1985. The relationship of plant size to self-pollination on Mertensia ciliata. Ecology 66(3):762-772.

GIBBS, P.E., LEWIS, G.P. \& LUGHADHA, E.N. 1999a. Fruit-set induced changes in the sex of flowers in Caesalpinia calycina (Leguminosae). Plant Biol. 1(6):665-669.

GIBBS, P.E., OLIVEIRA, P.E. \& BIANCHI, M.B. 1999b. Postzygotic control of selfing in Hymenaea stigonocarpa (Leguminosae - Caesalpinioideae), a bat-pollinated tree of the Brazilian cerrados. Int. J. Plant Science 160(1):72-78.

GIBBS, P.E. \& SASSAKI, R. 1998. Reproductive biology of Dalbergia miscolobium Benth. (Leguminosae-Papilionoideae) in SE Brazil: the effects of pistillate sorting on fruit-set. Ann. Bot. 81(6):735-740.

JACOBI, M.C., RAMALHO, M. \& SILVA, M. 2005. Pollination biology of the exotic rattleweed Crotalaria retusa L. (Fabaceae) in NE Brazil. Biotropica 37(3):357-363.
JUDD, W.S., CAMPBELL, C.S., KELLOGG, E.A., STEVENS, P.F. \& DONOGHUE, M.J. 2009. Sistemática vegetal: um enfoque filogenético. Artmed, Porto Alegre.

KEARNS, C.A., INOUYE, D.W. \& WASER, N.M. 1998. Endangered mutualisms: the conservation of plant-pollinator interactions. Ann. Rev. Ecol. Syst. 29:83-112.

LEWIS, G.P. \& GIBBS, P.E. 1999. Reproductive biology of Caesalpinia calycina and C. pluviosa (Leguminosae) of the caatinga of NE Brazil. Plant Syst. Evol. 217(1-2):43-53.

LIMA, H.C. 2000. Leguminosas arbóreas da Mata Atlântica: uma análise da riqueza, padrões de distribuição geográfica e similiaridades florísticas em remanescentes florestais do Estado do Rio de Janeiro. Tese de Doutorado, Universidade Federal do Rio de Janeiro, Rio de Janeiro.

LOPES, A.V. \& MACHADO, I.C. 1996. Biologia floral de Swartzia pickelii (Leguminosae - Papilionoideae e sua polinização por Eulaema spp. (Apidae-Euglossini). Rev. Bras. Bot. 19(1):17-24.

MACHADO, I.C. \& SAZIMA, M. 2008. Pollination and breeding system of Melochia tomentosa L. (Malvales), a keystone floral resource in the Caatinga: the role of Apis mellifera and autochthonous pollinators in fruit set. Flora 203(6):484-490.

MARTIN, F.W. 1959. Staining and observing pollen tubes in the style by means of fluorescence. Stain Technol. 34(3):125-128.

MITCHELL, R.J., KARRON, J.D., HOLMQUIST, K.G. \& BELL, J.M. 2004. The influence of Mimulus ringens floral display size on pollinator visitation. Func. Ecol. 18(1):116-124.

MORELLATO, L.P.C., TALORA, D.C., TAKAHASI, A., BENCKE, C.S.C., ROMERA, E.C. \& ZIPPARRO, V. 2000. Phenology of Atlantic rain forest trees: a comparative study. Biotropica 32(4):811-823.

NEWSTROM, L.E., FRANKIE, G.W. \& BAKER, H.G. 1994. A new classification for plant phenology based on flowering patterns in lowland Tropical Rain Forest trees at La Selva, Costa Rica. Biotropica 26(2):141-159.

NOGUEIRA, E.M.L. \& ARRUDA, V.L.V. 2006. Fenologia reprodutiva, polinização e sistema reprodutivo de Sophora tomentosa L. (Leguminosae - Papilionoideae) em restinga da praia da Joaquina, Florianópolis, sul do Brasil. Biotemas 19(2):29-36.

OPLER, P. 1983. Nectar production in a tropical ecosystem. In The biology of nectaries (B. Bentley \& T.S. Elias, eds). Columbia University Press, New York, p. 30-79.

PRATA DE ASSIS PIRES, J.P.A. \& FREITAS, L. 2008. Reproductive biology of two tree species of Leguminosae in a Montane Rain Forest in southeastern Brazil. Flora 203: 491-498

PANSARIN, L.M., PANSARIN, E.R. \& SAZIMA, M. 2008. Reproductive biology of Cyrtopodium polyphyllum (Orchidaceae): a Cyrtopodiinae pollinated by deceit. Plant Biol. 10(5):650-659.

PELLMYR, O. 2002. Pollination by animals. In Plant-animal Interactions an Evolutionary Approach (C.M. Herrera \& O. Pellmyr, eds) Blackwell Publishing, Oxford, p. 157-184.

PENNINGTON, R.T., STIRTON, C.H. \& SCHRIRE, B.D. 2005. Tribe Sophoreae. In Legumes of the World (G. Lewis, B.D. Schrire, B. Mackinder \& M. Lock, eds). Royal Botanic Gardens, Kew, p. 227-249.

PINHEIRO, M. \& SAZIMA, M. 2007. Visitantes florais e polinizadores de seis espécies arbóreas de Leguminosae melitófilas na Mata Atlântica no Sudeste do Brasil. Rev. Bras. Biociênc. 5(1): 447-449.

PRICE, P.W. 2002. Species interactions and the evolution of biodiversity. In Plant-animal Interactions an Evolutionary Approach (C.M. Herrera \& O. Pellmyr, eds) Blackwell Publishing, Oxford, p. 157-184.

PYKE, G.H. \& WASER, N.M. 1981. The production of diluted nectar by hummingbird and honeyeater flowers. Biotropica (13):260-270.

RADFORD, A.E., DICKINSON, W.C., MASSEY, J.R. \& BELL, C.R. 1974. Vascular plant systematics. Harper \& Row, New York.

RATHCKE, B. \& LACEY, E.P. 1985. Phenological patterns of terrestrial plants. Annu. Rev. Ecol. Syst. 16(2):179-214. 
ROMERA, E.C. 1999. Estudo fenológico em vegetação de duna do sudeste do Brasil. Dissertação de Mestrado, Universidade Estadual Paulista Júlio de Mesquita Filho, Rio Claro.

SAZIMA, I. \& SAZIMA, M. 1989. Mamangavas e irapuás (Hymenoptera, Apoidea): visitas, interações e conseqüências para a polinização do maracujá (Passifloraceae). Rev. Bras. Entomol. 33(1):109-118.

SAKAI, S., KATO, M. \& INOUE, T. 1999. Three pollination guilds and variation in floral characteristics of Bornean gingers (Zingiberaceae and Costaceae). Am. J. Bot 86(5):646-658.

SPRENT, J.I. 2001. Nodulation in legumes. Royal Botanic Gardens, Kew.

TORRES, C. \& GALETTO, L. 1998. Patterns and implications of floral nectar secretion, chemical composition, removal effects and standing crop in Mandevilla pentlandiana (Apocynaceae). Bot. J. Linn. Soc. 127(3):207-223.
Van WYK, B.E. 2005. Tribe Crotalarieae. In Legumes of the World (G. Lewis, B.D. Schrire, B. Mackinder \& M. Lock, eds). Royal Botanic Gardens, Kew, p. 273-281.

WESTERKAMP, C. 1997. Keel blossoms: bee flowers with adaptations against bees. Flora 192(2): 125-132.

WESTERKAMP, C. 2004. Ricochet pollination in cassias and how bees explain enantiostyly. Preliminary communication. In Solitary bees: conservation, rearing and management for pollination (B.M. Freitas \& J.O.P. Pereira, eds). MMA, Fortaleza.

ZIMMERMAN, M. 1988. Pollination biology of montane plants: relationship between rate of nectar production and standing crop. Am. Mid. Natur. 120(1):50-57.

Recebido em 05/10/09

Versão reformulada recebida em 16/03/10

Publicado em 30/03/10 